\title{
Deep Learning for Grain Size and Porosity Distributions Estimation on micro-CT Images
}

Fernando Bordignon ${ }^{\star 1}$, Leandro Passos de Figueiredo ${ }^{1}$, Rodrigo Exterkoetter ${ }^{1}$, Bruno Barbosa Rodrigues ${ }^{2}$ and Maury Duarte ${ }^{2},{ }^{1}$ LTrace Geophysical Solutions, ${ }^{2}$ PETROBRAS

Copyright 2019, SBGf - Sociedade Brasileira de Geofísica

This paper was prepared for presentation during the $16^{\text {th }}$ International Congress of the Brazilian Geophysical Society held in Rio de Janeiro, Brazil, 19-22 August 2019.

Contents of this paper were reviewed by the Technical Committee of the $16^{\text {th }}$ International Congress of the Brazilian Geophysical Society and do not necessarily represent any position of the SBGf, its officers or members. Electronic reproduction or storage of any part of this paper for commercial purposes without the written consent of the Brazilian Geophysical Society is prohibited.

Abstract

Digital rock physics uses numerical simulations of various processes to obtain properties of the rocks under study. The entire process is costly and computationally intensive. This abstract proposes a deep learning approach to estimate the grain size and porosity distributions from 3D grayscale micro tomography images. The goal is to overcome the high human and computational cost of segmentation and estimation of the aforementioned properties. 3D convolutional neural networks are trained with an idealized pack of spheres and two cementation models. An experiment on the Berea sandstone is performed, showing promising results for the technology.

\section{Introduction}

The main goal of digital rock physics (DRP) is to image and digitalize the rocks under study and then, numerically simulate physical processes to obtain the rock properties, such as permeability and elastic moduli (Andra, et al. 2013). Recently, the micro computed tomography (CT) analysis has gained interest because it can generate a 3D grayscale image representation of the rock, where the brightness is proportional to the CT-number of the material. The grayscale image is subjected to segmentation for labeling the pore and mineral phases presented at the image. The process of segmentation is semi-automatic, where algorithms are used to generate a segmentation which an experienced petrophysicist evaluates and tune the parameters to yield results that are as closest as possible to the reality (Sezgin and Sankur 2004, lassonov, Gebrenegus and Tuller 2009).

Porosity is one of the main properties used to characterize the rock under study. After the segmentation, one can easily compute the porosity by counting the pore labeled voxels and divide them by the total number of voxels. The grain size distribution (GSD) is another property which is used to characterize the hydrodynamic behavior of porous rocks (Rabbani and Ayatollahi 2015). The process of determining the grain size distribution involves analyzing the data via 3D segmentation algorithms that need to take into consideration the size and shape of grains, separating two or more cemented grains and determining their equivalent diameter.
The two properties of interest mentioned are heavily affected by the segmentation performed. Furthermore, they depend on user experience and trial and error iterations of the workflows to perform quality control on the results.

This work proposes a deep learning-based approach to overcome the segmentation and processing steps for determining the grain size and porosity distributions of the rock micro CT sample. Synthetic idealized rock models are generated to train a 3D convolutional neural network (CNN) (Ji, et al. 2013) with their respective grain size and porosity as the targets. The effectiveness of the method is tested on the Berea sandstone provided by (Andra, et al. 2013), yielding promising results for the technology. The next section details the methodology, followed by the experiment and conclusions.

\section{Method}

The main idea behind the methodology is to use synthetic idealized rocks to train the convolutional neural network and later apply the inference phase to real micro CT samples. The most common idealized rock model in the literature is the pack of spheres (Andra, et al. 2013, Mindlin 1949), which we can easily generate synthetic models and control the properties such as porosity and grain size. Furthermore, a sphere is a reasonable approximation of grains when working with grain size and porosity (Mavko, Mukerji and Dvorkin 2009).

\section{Synthetic data generation}

The training dataset was created using the sphere pack generation algorithm from (Baranau and Tallarek 2014) as a basis for the synthetic without cementation. A monodisperse pack of spheres is generated with approximately 10 thousand particles and radii equal to 50 inside a pack of size $1000 \times 1000 \times 1000$. This pack has a final porosity of 0.355 and, as the radii of all spheres are equal to 50 , the grain size distribution is a delta function at 50 . Figure 1 shows a slice of the 3D pack of spheres.

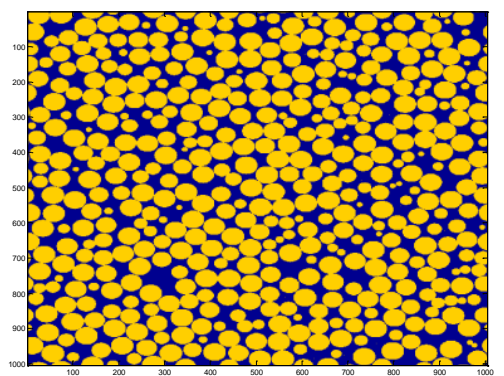

Figure 1: 2D slice of the 3D pack of spheres. 
To this base image is attributed a voxel size in millimeters matching the voxel size of the micro CT sample, therefore, each sphere will have a fixed size in millimeters which will be used as the grain size. For example, if the voxel size is $0.001 \mathrm{~mm}$ then the grain size is $0.05 \mathrm{~mm}$. To cover a range of grain sizes, the 3D image is rescaled $g$ times while maintaining the voxel size in millimeters, typically $g=5$. This results in the same number of 3D images with respective varying grain sizes. Ideally, the minimum and maximum grain sizes encompass the range reported at the literature for the rock under study.

At this step there are $g$ images with the same porosity of 0.355 . Therefore, to provide variability in porosity, we simulated different cementation levels based on the stiff and soft sand models (Mavko, Mukerji and Dvorkin 2009, Dvorkin, Gutierrez and Grana 2014). The soft-sand model considers cementation away from the grain contacts, whereas the stiff-sand model adds cementation at grain contacts. Hence, $p$ levels of porosity are chosen covering a wide range from typically 0.05 to the porosity of the original pack of spheres.

Using each level of porosity for each scaled image, the soft-sand model is simulated by randomly flipping the necessary number of pore voxels, away at least 1 voxel from the grains, to reach the porosity level. Similarly, the stiff-sand model is simulated by using Truncated Gaussian Simulation (Armstrong, et al. 2003) with an unconstrained geostatistical simulation method such as FFT-MA (Le Ravalec, Noetinger and Y. Hu 2000) or SGS (Deutsch and Journel 1998). We use a spherical variogram of range equal to half of the grain size and variance equal to one. The simulation is binarized using the following threshold $l$ :

$$
l=C^{-1}\left(1-\Phi_{i} / \Phi_{p}\right),
$$

where $C^{-1}$ is the inverse normal cumulative function, $\Phi_{i}$ is the desired porosity level and $\Phi_{p}$ is the porosity of the sphere pack. Figure 2 shows examples of $2 \mathrm{D}$ slices from the 3D images for 3 levels of porosity and grain sizes.

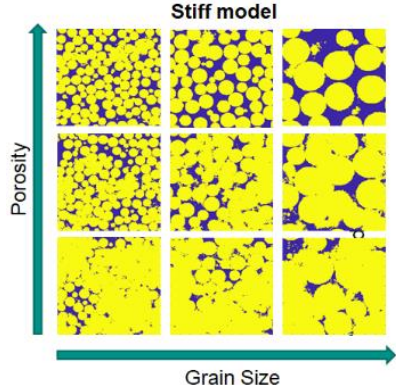

Grain Size

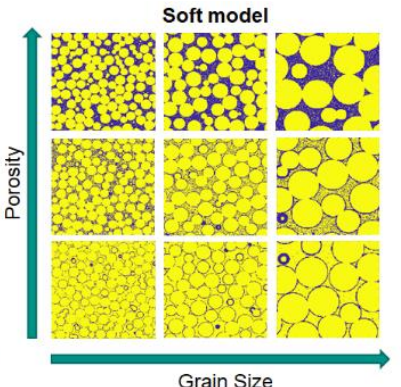

Grain Size
Figure 2: Slices from 3D examples of the two types of cementation, levels of porosity and grain sizes.

Up to this stage, there are $2 p g$ binary images, the next step is to simulate the CT imaging. Hence, the real CT image is used to calculate a filter that should map the binary images to the grayscale CT. We propose approximating the imaging process by a linear system, therefore the first step in calculating the filter is to apply a simple threshold segmentation to the real CT image and define the mean value for the pore and grain phases of the rock. Figure 3 shows the histogram with the values indicated.

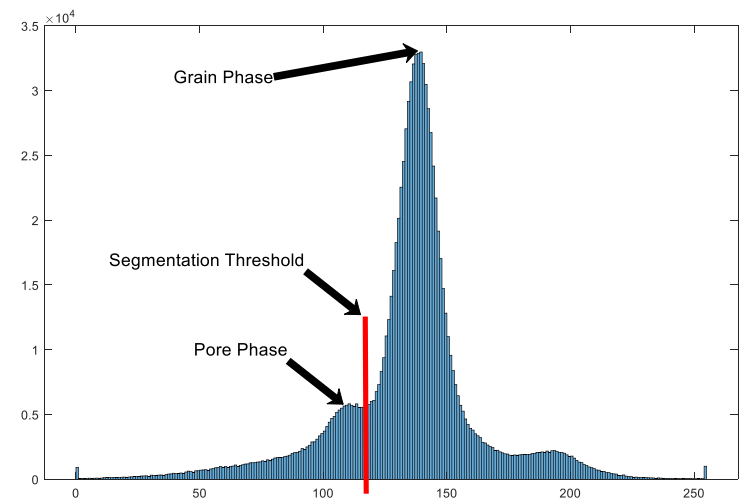

Figure 3: Histogram of the CT image with the two main phases and segmentation threshold indicated.

With the real CT image and the segmented one, we apply the grain phase value to the voxels with value 1 and the pore phase value to the voxels with 0 at the segmented image. Thus, the filter is calculated by creating a linear system of equations $A x=b$, where $x$ is the filter with a size of typically 50 to 80 coefficients, $b$ is a single $1 \mathrm{D}$ vector extracted from the original CT image and $A$ is the convolutional matrix with constructed with the same 1D vector position of $b$ from the segmented image with the grain-pore phase values. Solving the linear system gives us the $x$ filter that maps, using convolution, the grain-pore phases mean value segmented images to the original CT image frequency content.

Subsequently, we use the 3D synthetic images, generated at the previous steps, to map the 1-valued voxels to the grain phase mean value from the original CT image and the 0 -valued voxels to the pore phase mean value. Next, a white noise is applied after the mapping to simulate the noise present at the CT image. The $x$ filter is then applied to the modified images generating a set of approximated synthetic grayscale CT images.

At the end of the synthetic image generation, there will by $2 p g$ synthetic binary segmented images that have each one a grain size and porosity label, along with $2 p g$ respective synthetic approximations of real CT images in grayscale. The step-by-step of the synthetic generation is summarized below:

1. Generate a random sphere pack of monodisperse spheres.

2. Assign the voxel size for the sphere pack equal to the voxel size of the intended CT image to analyze.

3. Rescale the sphere pack for covering the grain sizes according to the range reported in the literature.

4. Add soft cementation by randomly flipping 0 -valued voxels to reach the porosity levels stipulated

5. Add stiff cementation using a geostatistical simulation

6. Segment the original CT image using a simple threshold and determine the mean values for the pore and grain phases.

7. Calculate the CT filter $x$

8. Map the generated binary synthetic images to the mean values of pore and grain phases and add white noise.

9. Filter the mapped images with the CT filter $x$ 
With the synthetic 3D binary and grayscale image pairs ready, the next step is to sample a small $3 D$ window to use it as input to the convolutional neural network. A number $s$ of samples per case is chosen, typically 400 to 500 . The total number of training samples is going to be $2 p g s$, where the grayscale image input samples are subsampled with a 3D window of size $w$, typically smaller than 128 .

The subsampling will result in a smaller 3D window that have 2 labels, the grain size, that depends only on the original synthetic image and the porosity, which changes locally for each window. Hence, the porosity label is recalculated using the binary respective subsample. Finally, the training dataset consists of $2 p g s$ 3D grayscale synthetic CT samples of $w^{3}$ voxels each and 2 labels as outputs (targets): the local window porosity and the grain size.

\section{Convolutional Neural Network}

The network used for learning the grain size and porosity distributions was the convolutional neural network due to it capabilities for spatial patterns recognition and identification, spatial features learning and nonlinear regression fitting ( $\mathrm{Ji}$, et al. 2013). Table 1 shows the summary of the network used, its size and the total number of parameters. All layers have 'relu' activations except for the last one that uses 'tanh'.

Table 1: Summary of the CNN.

\begin{tabular}{|r|c|c|r|r|}
\hline \multicolumn{1}{|c|}{$\#$} & Type & Stride & Neurons & Parameters \\
\hline 1 & Convolutional 3D & No & 16 & 448 \\
\hline 2 & Convolutional 3D & $2 \times 2 \times 2$ & 32 & 13,856 \\
\hline 3 & Dropout & - & 0 & 0 \\
\hline 4 & Convolutional 3D & No & 32 & 27,680 \\
\hline 5 & Convolutional 3D & $2 \times 2 \times 2$ & 64 & 55,360 \\
\hline 6 & Dropout & - & 0 & 0 \\
\hline 7 & Convolutional 3D & No & 64 & 110,656 \\
\hline 8 & Convolutional 3D & $2 \times 2 \times 2$ & 64 & 110,656 \\
\hline 9 & Dropout & - & 0 & 0 \\
\hline 10 & Convolutional 3D & No & 128 & 221,312 \\
\hline 11 & Convolutional 3D & No & 256 & 884,992 \\
\hline 12 & Convolutional 3D & No & 2 & 514 \\
\hline & Total: & 656 & $1,425,474$ \\
\hline
\end{tabular}

Popular layers for deep neural networks include pooling layers (Ji, et al. 2013), which decreases the size of the network by pooling the maximum activation of the previous layer, gaining in invariance to certain transformations of the input but compromising on precision. These types of layers were not used in our model due to the need of precise information about the grain and cementation shapes to perform the task of predicting porosity and grain size, which is a type of regression. Another popular layer often employed is the dropout, which is included in our model to prevent overfitting. This layer randomly drops to zero a percentage of the activations of the previous layer, in this case $20 \%$.

The input layer is sized according to $w$, therefore we've chosen to use $w=64$. This can be changed as needed depending on the case. A recommendation is to choose a window size that fits at least a few average-sized grains, so that the CNN can properly infer the outputs not only by the grayscale values itself, but by using also the shape of the grains and pores.

\section{Experiment}

\section{Dataset}

The methodology was tested using the Berea sandstone micro CT by (Andra, et al. 2013). The 3D image has a total of $1024^{3}$ pixels with a voxel edge length of $0.74 \mu \mathrm{m}$. As the image has extraordinary resolution, it was rescaled to $512^{3}$ and converted to grayscale $[0,255]$ for this experiment, thus, saving memory and not compromising the ability to identify clearly the grains and pores in the image. This rescaled and normalized image will be referred form now on as original CT image. Figure 4 shows a 2D slice of the rescaled image.

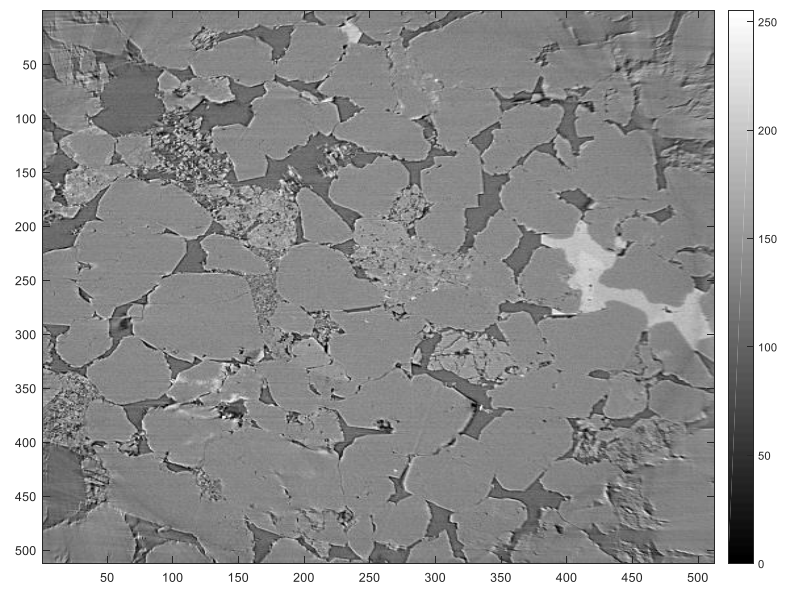

Figure 4: Slice of the Berea sandstone sample with a voxel edge of $1.48 \mu \mathrm{m}$.

From visual inspection, it was determined that the grains have a maximum cross section range of $0.03 \mathrm{~mm}$ to $0.12 \mathrm{~mm}$. This range is not the real $3 \mathrm{D}$ grain size range distribution, but is a broad range that should cover the real one. Therefore, the synthetic data generation starts with rescaling the pack of spheres to match the grain size range, in this case, the rescale factors were set to $(0.4,0.7,1,1.3,1.6)$. Hence, resulting in a $g=5$ and grain sizes of $(0.0296,0.0518,0.0740,0.0962,0.1184)$ in $\mathrm{mm}$.

Literature on the Berea sandstone states that the porosity ranges from $19 \%$ to $26 \%$ (Churcher, et al. 1991), in fact, the sample under study has a porosity of $20 \%$ (Andra, et al. 2013). Therefore, the porosity levels for the synthetic data is set to $(0.005,0.086,0.167,0.248,0.330)$, yielding $p=5$ and $2 p g=503 \mathrm{D}$ binary training images.

At this stage, the computation of the CT filter is performed. The first step is to select the grain and pore phases mean values using the histogram. Figure 3 shows the histogram of the Berea sandstone. The grain phase value was set to 135 , the pore to 108 and the segmentation threshold to 115. A new segmented image is generated by applying the segmentation threshold to the original CT image, setting to 108 all the voxels with values below 115 and to 135 all voxels with values above 115 .

Next, we select a single 1D vector position to estimate the filter using a linear system. The convolution matrix $A$ is constructed with the 1D vector from the segmented twophase valued image and $b$ is equal to the $1 \mathrm{D}$ vector from 
the original image. Solving the system yields the filter $x$, which is shown in Figure 5.

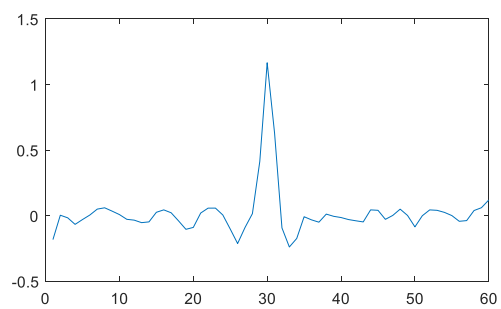

Figure 5: Filter calculated for mapping the segmented images to the $\mathrm{CT}$ frequency spectrum.

A new set of 50 images is created by mapping the 0 -valued voxels of the binary synthetic images to 108 and the 1 valued voxels to 135 . At last, the images are convolved with the computed filter, producing the final set of 50 grayscale images.

To generate the final training dataset an $s=400$ is chosen. For each 50 binary $0 / 1$ synthetic images and their grayscale filtered counterparts, $s$ smaller 3D windows of size $w=64$ are randomly sampled from the two images at the same time. The grayscale windowed image is added to the input training set and labeled with: the respective grain size from the image that originated it; and the porosity label calculated from the binary $0 / 1$ windowed synthetic.

Finally, the training dataset has inputs with 20,000 images of $64 \times 64 \times 64$ voxels and outputs (targets) with 20,000 two dimensional labels, containing grain size and local window porosity.

\section{Training and Results}

The convolutional neural network presented at the methodology section was trained with the "adam" optimizer and 1,000 epochs (iterations over the training batch). As the training data is entirely synthetic, there is no point in separating the data into training, test and validation, because any random split will result in testing and validation sets with very similar samples as the training. Normalization was performed to maintain the inputs and outputs inside the range $[-0.9,0.9]$. The network converged to a low error, showed at Figure 6.

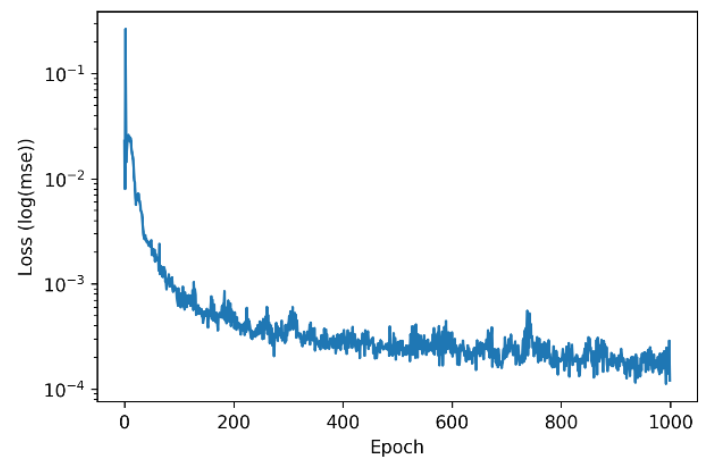

Figure 6: Training loss in log scale over the epochs.

Keras framework was used with TensorFlow backend, along with a GTX1080Ti GPU, where the training consumed 660s. The convergence of training is evidentiated by the similarity of histograms of grain size and porosity between the training targets set and the results when the training inputs are fed to the network. Figure 7 shows the comparison aforementioned.

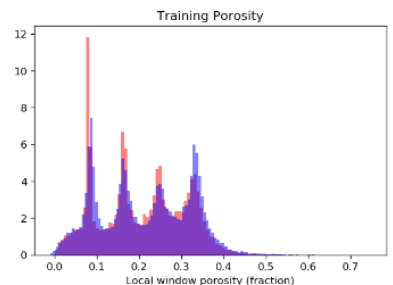

(a)

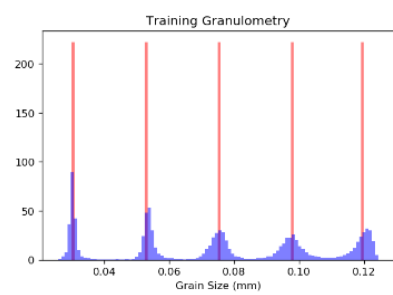

(b)
Figure 7: Training targets (red) compared to the results of the CNN when applying the input training data (blue) for (a) porosity and (b) grain size.

The inference phase was performed by sampling the original Berea sandstone $512^{3}$ CT grayscale image 5,000 times using the $64^{3}$ window. Therefore, the result will be porosity and grain size distributions estimation, due to the variability of those properties inside the same CT sample.

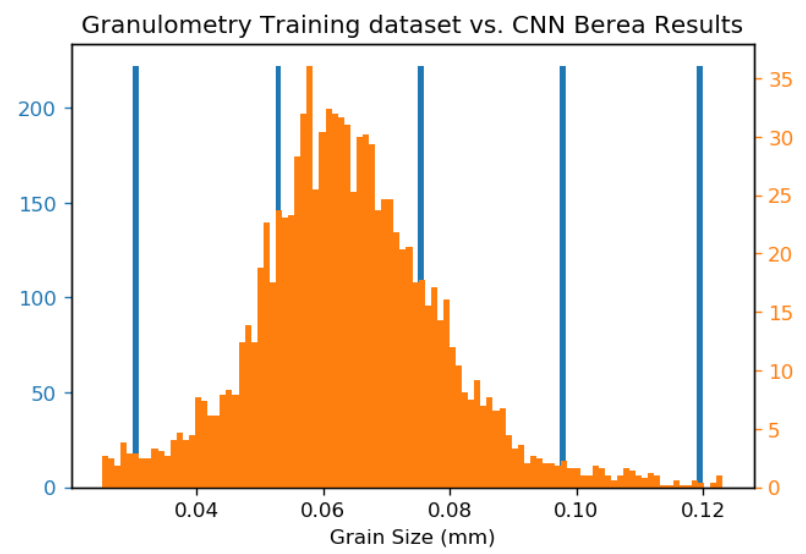

Figure 8: Grain size distribution result from the CNN applied to the Berea sandstone (orange) and training targets (blue).

Figure 8 shows the granulometry distribution results from the CNN compared to the training data. The average grain size was $0.064 \mathrm{~mm}$ and the standard deviation $0.015 \mathrm{~mm}$. It is possible to notice that, even though the grain sizes provided to the network as training inputs are a set of fixed sizes, the CNN was able to interpolate and combine different activation levels, approximating what is reported at the literature (Churcher, et al. 1991) and compatible with visual inspection.

Figure 9 compares the results of the CNN for porosity with the porosity extracted from a segmentation available with the original dataset (Andra, et al. 2013). The match between the CNN results and the reference segmentation is evident. 


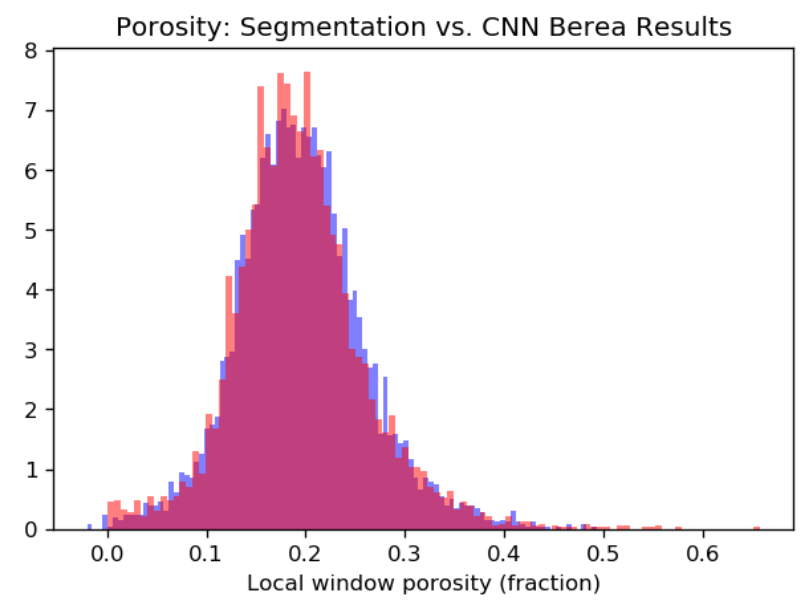

Figure 9: Porosity result of the CNN in blue compared to the reference segmentation in red.

\section{Discussion}

The methodology proposed harness the idea of using synthetic data to train a deep neural network, aiming to apply it to real data. The goal was to balance the compromise between mimicking the real rock characteristics by using idealized rock models and maintaining a complexity level that still allows for analytical simple metrics to be extracted from the training dataset. In other words, to get as close as possible to reality with synthetic controlled data.

The method achieved an excellent result for porosity, proven by the comparison with a porosity distribution extracted from a reference segmentation. Also, the granulometry distribution is in line with the reports from the literature, although a more comprehensive comparison is needed, perhaps using an algorithmic method for estimating $3 \mathrm{D}$ grain size distributions, as in (VAN Dalen and Koster 2012).

One caveat of the methodology is the estimation of the CT filter to map the synthetic segmented images to the real CT frequency spectrum, as there is the need to segment the real CT image. Although, the segmentation can be a simple one, without much rigor, due to the robustness of the CNNs. Even with a poor map from the segmented synthetic to the real CT, the network is expected to perform in a similar manner. Adding noise also helps by increasing in the robustness of the CNN. An alternative to estimating the filter by segmentation is to estimate the frequency content of the real CT image and use a simple bandpass filter at the synthetic segmented. The filter used was 1D, another improvement would be to calculate a $3 \mathrm{D}$ filter assuming isotropy.

\section{Conclusion}

Considering the CNNs capacity for spatial patterns identification, the application of deep learning to micro CT samples is promising. The results are promising for porosity and grain size distributions estimations. Future work should investigate the use of rock physics models to include petrophysical properties at the estimation, such as bulk modulus. Additionally, a more general training is also needed to improve the applicability of a trained network to different types of rocks, including carbonates.

\section{Acknowledgments}

The authors would like to acknowledge PETROBRAS for the availability and technical support during the development of this work.

\section{References}

Andra, Heiko, et al. "Digital rock physics benchmarksPart I: Imaging and segmentation." Computers \& Geosciences, 2013: 25-32.

Armstrong, M., et al. Plurigaussian simulations in geosciences. Springer, 2003.

Baranau, Vasili, and Ulrich Tallarek. "Random-close packing limits for monodisperse and polydisperse hard spheres." Soft Matter, 2014: 3826-3841.

Blunt, M, B Bijeljic, A Raeini, and P Mostaghimi. Pore Scale Modelling. 2018. http://www.imperial.ac.uk/earthscience/research/researchgroups/perm/research/pore-scalemodelling/micro-ct-images-and-networks/bereasandstone/ (accessed 2018).

Churcher, P. L., P French, J Shaw, and L Schramm. "Rock Properties of Berea Sandstone, Baker Dolomite, and Indiana Limestone." SPE International Symposium on Oilfield Chemistry. Anaheim: Society of Petroleum Engineers, 1991. 431-446.

Delle Piane, Claudio, and Joel Sarout. "Effects of water and supercritical $\mathrm{CO} 2$ on the mechanical and elastic properties of Berea sandstone." International Journal of Greenhouse Gas Control, 2016: 209-220.

Deutsch, C.V., and A.G. Journel. GSLIB: Geostatistical Software Library and User's Guide, second edition. Oxford: Oxford University Press, 1998.

Dvorkin, J., M. A. Gutierrez, and D. Grana. Seismic Reflections of Rock Properties. Cambridge University Press, 2014.

lassonov, P., T. Gebrenegus, and M. Tuller. Segmentation of $X$-ray computed tomography images of porous materials: a crucial step for characterization and quantitative analysis of pore structures. Water Resources Research, 2009.

Ji, S, W Xu, M Yang, and K Yu. "3D Convolutional Neural Networks for Human Action Recognition." IEEE Transactions on Pattern Analysis and Machine Intelligence 35, no. 1 (2013): 221-231.

Le Ravalec, Mickaële, Benoît Noetinger, and Lin Y. Hu. "The FFT Moving Average (FFT-MA) Generator: An Efficient Numerical Method for Generating and Conditioning Gaussian Simulations." Mathematical Geology, 2000: 701-723.

Mavko, Gary, Tapan Mukerji, and Jack Dvorkin. The Rock Physics Handbook. Cambridge University Press, 2009.

Mindlin, R. D. Compliance of elastic bodies in contact. J. Appl. Mech., 1949.

Rabbani, Arash, and Shahab Ayatollahi. "Comparing three Image Processing Algorithms to Estimate 
the Grain-Size Distribution of Porous Rocks from Binary 2D Images and Sensitivity Analysis of the Grain Overlapping Degree." Special Topics \& Reviews in Porous Media: An International Journal, 2015: 71-89.

Sezgin, M., and B. Sankur. Survey over image thresholding techniques and quantitative performance evaluation. Journal of Electronic Imaging, 2004

VAN Dalen, Gerard, and Martin Koster. "2D \& 3D particle size analysis of micro-CT images." BrukermicroCT User Meeting. Brussels, 2012. 157-171.

Weibel, Ewald, and Brian Ralph. "Stereology 4." Journal of Microscopy, 1976: 209. 